\title{
Nicotinamide attenuates the decrease in dendritic spine density in hippocampal primary neurons from 5xFAD mice, an Alzheimer's disease animal model
}

\author{
Hyunju Kim ${ }^{1,2+}$, Bora Kim ${ }^{3,4+}$, Hye-Sun Kim ${ }^{1,2,5,6^{*}}$ (1) and Joo-Youn Cho ${ }^{3,7^{*}}$
}

\begin{abstract}
Alzheimer's disease (AD) is the most common neurodegenerative disease characterized by memory loss and the presence of amyloid plaques and neurofibrillary tangles in the patients' brains. In this study, we investigated the alterations in metabolite profiles of the hippocampal tissues from 6, 8, and 12 month-old wild-type (WT) and 5xfamiliar AD (5xFAD) mice, an AD mouse model harboring 5 early-onset familiar AD mutations, which shows memory loss from approximately 5 months of age, by exploiting the untargeted metabolomics profiling. We found that nicotinamide and adenosine monophosphate levels have been significantly decreased while lysophosphatidylcholine (LysoPC) (16:0), LysoPC (18:0), and lysophosphatidylethanolamine (LysoPE) (16:0) levels have been significantly increased in the hippocampi from 5xFAD mice at 8 months or 12 months of age, compared to those from age-matched wild-type mice. In the present study, we focused on the role of nicotinamide and examined if replenishment of nicotinamide exerts attenuating effects on the reduction in dendritic spine density in hippocampal primary neurons from 5xFAD mice. Treatment with nicotinamide attenuated the deficits in spine density in the hippocampal primary neurons derived from 5xFAD mice, indicating a potential role of nicotinamide in the pathogenesis of AD. Taken together, these findings suggest that the decreased hippocampal nicotinamide level could be linked with AD pathogenesis and be a useful therapeutic target for AD.
\end{abstract}

Keywords: Alzheimer's disease, Dendritic spine density, Hippocampus, Metabolomics, Nicotinamide

\section{Introduction}

Alzheimer's disease (AD) animal models have been used to investigate pathogenic mechanisms, discover potential biomarkers, and evaluate novel treatments $[1,2]$. The 5xFAD mouse, a widely used AD mouse model, has 5 familiar AD-linked mutations, i.e., the Swedish (K670 N/ M671 L), Florida (I716V), and London (V717I) mutations in amyloid precursor protein (APP) and the M146 $\mathrm{L}$ and L286 V mutations in presenilin-1 (PSEN1) [2]. $5 x F A D$ mice, characterized by cerebral amyloid plaques

\footnotetext{
*Correspondence: hyisun@snu.ac.kr; joocho@snu.ac.kr

${ }^{\dagger}$ Hyunju Kim and Bora Kim contributed equally to this work.

'Department of Pharmacology, College of Medicine, Seoul National

University, 103 Daehakro, Jongro-gu, Seoul, Republic of Korea

${ }^{3}$ Department of Clinical Pharmacology and Therapeutics, College of

Medicine, Seoul National University, 103 Daehakro, Jongro-gu, Seoul,

Republic of Korea

Full list of author information is available at the end of the article
}

and gliosis, show massive $A \beta_{1-42}$ burdens from 2 months of age, declined synaptic markers from 4 months of age, and cognitive impairment from approximately 5 months of age [2-5]. Thus, pathological mechanisms of $A D$ could be evaluated by analyzing biochemical changes in the brain in 5xFAD mice at different disease stages.

Altered metabolites reflect downstream changes of genomic, transcriptomic, and proteomic fluctuations, and metabolomics data, such as an accurate biochemical profile, can thus be used to visualize and interpret complex biological networks of AD. Multiple human studies have focused on metabolomics analysis of blood-derived samples, cerebrospinal fluid, and postmortem brain tissues, since, unlike for mouse models, premortem brain samples are not available [6]. Targeted metabolomics of 44 postmortem brain samples showed that a panel of sphingolipids is associated with the severity of $\mathrm{AD}$

(C) The Author(s). 2020 Open Access This article is distributed under the terms of the Creative Commons Attribution 4.0 International License (http://creativecommons.org/licenses/by/4.0/), which permits unrestricted use, distribution, and 
pathology [7-9]. Arginine metabolism is altered in the postmortem hippocampus, superior frontal gyrus, and cerebellum of $\mathrm{AD}$ patients and normal control subjects [10].

Brain metabolic perturbations have been described in several transgenic AD models. For instance, the disturbances in metabolites of the glycolytic pathway (glucose6-phosphate and glycerol-3-phosphate) and tricarboxylic acid (TCA) cycle ( $\alpha$-ketoglutarate, fumarate, and succinate) were identified in astrocytes derived from newborn $5 \times$ FAD mice [11], and pantethine treatment reduced the extent of metabolic perturbation and decreased the inflammatory processes in these astrocytes, indicating the role of altered brain energetics in the $\mathrm{AD}$ pathogenesis; metabolic profile analyses revealed region-specific metabolic changes in the hippocampus, cortex, cerebellum, and olfactory bulbs in APP/PS1 mice [12, 13], and metabolomics signatures, including mitochondrial dysfunction and altered energy metabolism indicated by changes in nucleotide, TCA cycle, energy transfer, neurotransmitter, and amino acid metabolic pathways, were identified in APP/PS1 mice [14]; in addition, significant changes in metabolite compositions, including accumulation of fatty acids, alterations in phospholipids and acylcarnitines related to neural membrane degradation, and impaired energy management, were observed in the hippocampus and cortex in APP/PS1 mice [13]. Because the metabolic pathways are conserved through evolution $[15,16]$, the metabolic signatures identified in $\mathrm{AD}$ mouse models could be directly translated into human studies [17]. Therefore, metabolomics screening in transgenic models could be useful for the understanding of the pathological mechanisms of AD.

Amyloid $\beta$ peptide $(A \beta)$ deposition and neurofibrillary tangles in the $\mathrm{AD}$ hippocampus, which is the central brain region to exhibit neurodegeneration and other AD-related alterations, possibly lead to cognitive impairment $[18,19]$. In addition, hippocampal oxidative stress is implicated in neurodegenerative diseases and neurodevelopmental disorders $[20,21]$. However, no study has investigated the metabolomics profiling of the hippocampus in the 5xFAD mouse model. This study aims to apply an untargeted metabolomics approach to characterize metabolic abnormalities in the hippocampus in 5xFAD mice at different $\mathrm{AD}$ progression stages.

\section{Materials and methods}

\section{Experimental animals}

All experimental procedures were approved by the Animal Care Committee of Seoul National University (Approval Number: SNU-131016-1). Transgenic mice with 5 familial AD mutations were purchased from Jackson Laboratories (strain: B6SJL-Tg [APPSwFlLon, PS1*M146 L"L286 V] 6799Vas/J) and were bred by crossing hemizygous transgenic male mice with B6SJL F1 female mice.
Male WT and 5xFAD mice were used in all experiments. Animal treatment and maintenance were performed in accordance with the Institutional Animal Care and Use Committee Guidelines of Seoul National University, Seoul, Korea.

\section{Chemicals and reagents}

High-performance liquid chromatography grade solvents, including methanol, acetonitrile, and water, were purchased from J.T. Baker (PA, USA). Formic acid, nicotinamide, adenosine monophosphate, LysoPC, and LysoPE were obtained from Sigma-Aldrich (MO, USA) and Avanti Polar Lipids (AL, USA).

\section{Hippocampus sample preparation for metabolomics}

The hippocampus was weighed ( $20 \mathrm{mg}$ wet), homogenized in methanol: water (4:1, v/v; $50 \mu \mathrm{l} / \mathrm{mg}$ tissue), and frozen in liquid nitrogen for $1 \mathrm{~min}$. The homogenate was thawed at room temperature and was then sonicated for $5 \mathrm{~s}$. After adding acetonitrile ( $30 \mu \mathrm{l} / \mathrm{mg}$ tissue), the homogenate was vortexed for $5 \mathrm{~s}$ incubated for $1 \mathrm{~h}$ at $-20^{\circ} \mathrm{C}$, and centrifuged at $13,000 \mathrm{rpm}$ for $15 \mathrm{~min}$ at $4{ }^{\circ} \mathrm{C}$. The pellet was reconstituted in radioimmunoprecipitation assay buffer (Elpis-Biotech, Daejeon, Korea), and the concentration of total protein was determined using the Pierce BCA Protein Assay Kit (Thermo Scientific, MA, USA). The supernatant was transferred into a microcentrifuge tube and was dried under an N2 evaporator. The dry extracts were then reconstituted with different volumes of solvent mixtures (acetonitrile: $\mathrm{H}_{2} \mathrm{O}, 1: 1, \mathrm{v} / \mathrm{v}$ ) based on the sample's protein levels, and the mixtures were sonicated for $10 \mathrm{~min}$ and centrifuged at $14,000 \mathrm{rpm}$ for $15 \mathrm{~min}$ at $4{ }^{\circ} \mathrm{C}$ to remove insoluble debris. The supernatant was used for the LC-MS analysis.

\section{Untargeted metabolomics}

A $4 \mu \mathrm{l}$ aliquot of the sample was injected into a Waters UPLC system with a reverse phase $2.1 \times 100 \mathrm{~mm}$ ACQUITY $1.8 \mu \mathrm{m}$ HSS T3 column. The gradient mobile phase comprised $0.1 \%$ formic acid (Solution A) and methanol containing $0.1 \%$ formic acid (Solution B). Each sample was resolved for $20 \mathrm{~min}$ at a flow rate of $0.4 \mathrm{ml} /$ min. The gradient consisted of 5\% Solution B for $1 \mathrm{~min}$, 5-30\% Solution B over 1-8 min, 30-70\% Solution B over $8-13 \mathrm{~min}$, and $95 \%$ Solution B for $14 \mathrm{~min}$ (maintaining for $2 \mathrm{~min}$ ). The samples were equilibrated in $95 \%$ Solution A for 3.5 min before injection. A Waters Xevo G2 time-of-flight mass spectrometry was operated in positive and negative ionization modes. To obtain consistent differential variables, we prepared a pooled sample (quality control [QC] sample) by mixing aliquots of individual samples. Replicates of the QC sample were acquired in a series of injections, and data were obtained by random injection. The metabolomic dataset was 
deconvoluted and peak aligned using Progenesis QI software (version 2.3, Nonlinear Dynamics, Newcastle, UK). The most suitable candidate QC sample was chosen by the highest similarity using Progenesis QI software. Vector alignment quality was manually processed, and files were aligned with sensitivity $(10 \mathrm{ppm})$, retention time limits, and peak normalization (normalization to all compounds) at the default values. Subsequently, ions with a \% CV of abundance $>30$ in the QC were removed. A significantly differential expression was defined as a false discovery rate (FDR) adjusted $p$-value $(q$-value $)<0.05$. The FDR was obtained by adjusting the raw $p$-values of the $t$-test using the method of Benjamini and Hochberg [22].

\section{Hippocampal primary neuron culture}

Hippocampal primary neurons, which were prepared from postnatal day 1 to 2 xxFAD mice by dissociating with $0.25 \%$ trypsin, were plated onto coverslips coated with poly-L-lysine (Sigma, St Louis, USA). Neurons were grown in Neurobasal medium (Gibco, CA, USA) supplemented with B27 (Gibco, CA, USA), 2 mM GlutaMAX-I (Gibco, CA, USA), and $100 \mu \mathrm{g} / \mathrm{ml}$ penicillin/streptomycin (Gibco, CA, USA) at $37^{\circ} \mathrm{C}$ in a humidified environment of $95 \% \mathrm{O}_{2} / 5 \% \mathrm{CO}_{2}$.

\section{Dendritic spine density analysis}

Hippocampal primary neurons were transfected with $6 \mu \mathrm{g}$ CAG-IRES-mGFP plasmid (a generous gift from Dr.
Kolodkin) in 18-mm glass coverslips in 60-mm dishes. Nicotinamide (Sigma, St Louis, USA) was prepared in saline. Neurons were treated with nicotinamide or vehicle for $24 \mathrm{~h}$. The number of dendritic spines was evaluated at 18-19 days in vitro (DIV). Fluorescent images were acquired using a confocal microscope (LSM 510; Carl Zeiss, Jena, Germany) using the same setting condition for all samples. Spines were counted within the 20$\mu \mathrm{m}$ to $50-\mu \mathrm{m}$ segments on secondary dendrites extending $50-100 \mu \mathrm{m}$ beyond the soma.

\section{Statistical analysis}

One-way ANOVA and independent $t$-test (SPSS, IL, USA) were used for determining the statistical significance. A $p<0.05$ or $q<0.05$ was considered statistical significance.

\section{Results}

\section{Hippocampus metabolic profiling}

Untargeted metabolomics profiling of hippocampal tissues was performed in WT and $5 x F A D$ mice at three different stages (6, 8, and 12 months) (Fig. 1 a). The number of WT and 5xFAD mice and the weights of hippocampal tissues used are described in Table 1. In total, 2950 compound ions were identified in the positive ion and negative ion electrospray ionization $\mathrm{ESI}^{+}$and $\mathrm{ESI}^{-}$, respectively) modes. We selected ions with a $q$-value of less than 0.05 in $5 x F A D$ mice at each stage. Notably, 51 and 115 features were significantly different between a
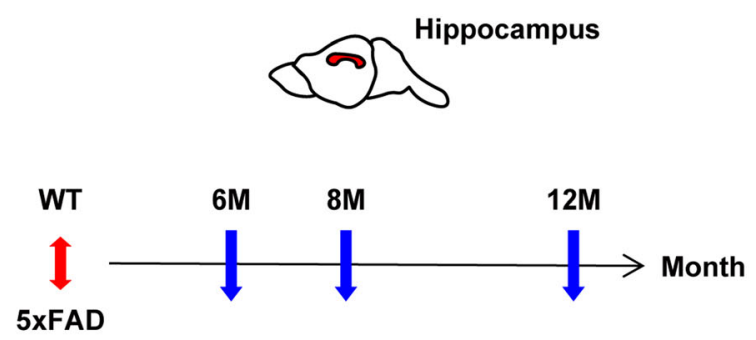

b

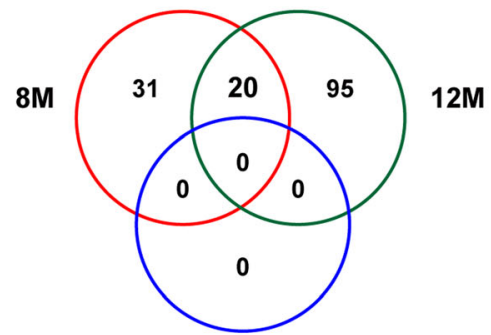

$6 \mathrm{M}$
C

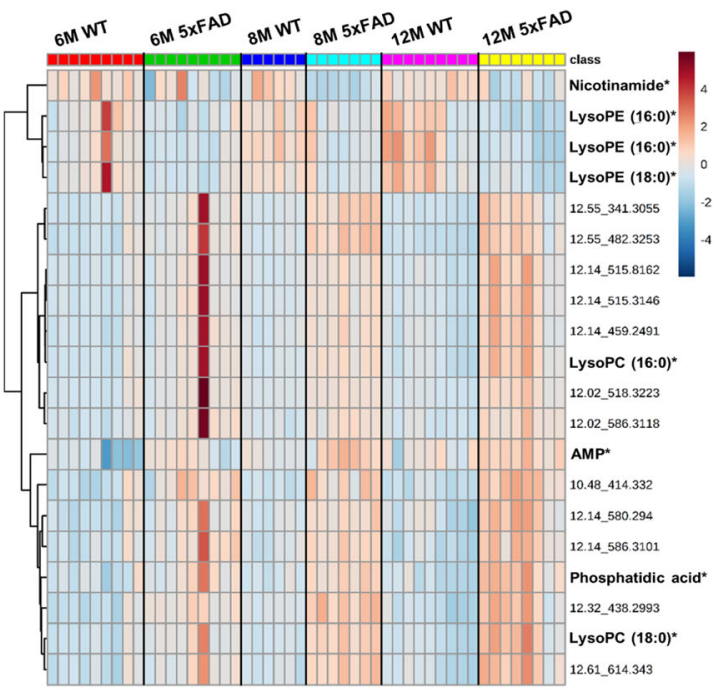

Fig. 1 Hippocampal metabolomics of 5XFAD mice at different disease progression stages. a Time course of the hippocampal sample collection. $\mathbf{b}$ Venn diagram representing overlapping ion features that were significantly different between the hippocampi of WT and 5xFAD mice $(q<0.05)$ at 6,8 , or 12 months of age. c Hierarchically clustered heat map of the relative intensity of 20 metabolic markers. Rows and columns represent the individual mice and the 20 selected metabolites (retention time_m/z, ${ }^{*}$ identified or putative metabolites), respectively. Each cell is colored based on the relative intensity 
Table 1 The number of male mice and tissue weights. The mice were classified into 6 groups based on age $(6,8$, and 12 months). HPC, hippocampus; SD, standard deviation

\begin{tabular}{|c|c|c|}
\hline \multirow{3}{*}{$\begin{array}{l}\text { Age } \\
\text { (month) }\end{array}$} & \multirow[t]{3}{*}{ Genotype } & \multirow{3}{*}{$\begin{array}{l}\mathrm{HPC} \text { weight }(\mathrm{mg}) \\
\text { Mean } \pm \mathrm{SD} \\
\text { (min-max) }\end{array}$} \\
\hline & & \\
\hline & & \\
\hline \multirow[t]{4}{*}{6} & \multirow[t]{2}{*}{ WT } & $20.5 \pm 3.4$ \\
\hline & & $(15.0-25.6)$ \\
\hline & \multirow[t]{2}{*}{$5 x F A D$} & $20.3 \pm 5.4$ \\
\hline & & $(14.5-28.6)$ \\
\hline \multirow[t]{4}{*}{8} & \multirow[t]{2}{*}{ WT } & $21.1 \pm 2.7$ \\
\hline & & $(17.8-25.7)$ \\
\hline & \multirow[t]{2}{*}{$5 \times F A D$} & $24.4 \pm 3.2$ \\
\hline & & $(20.4-29.2)$ \\
\hline \multirow[t]{4}{*}{12} & \multirow[t]{2}{*}{ WT } & $19.7 \pm 4.2$ \\
\hline & & $(10.0-24.9)$ \\
\hline & \multirow[t]{2}{*}{$5 x F A D$} & $18.4 \pm 2.8$ \\
\hline & & $(13.1-21.8)$ \\
\hline
\end{tabular}

WT and 5xFAD mice at 8 and 12 months of age, respectively (Fig. 1b). No markers differed between WT and 5xFAD mice at 6 months of age. Twenty ions that were significantly different between WT and 5xFAD mice at both 8 and 12 months of age were selected for further identification. The hierarchically clustered heat map illustrates significant differences in the relative intensity of the selected 20 markers (Fig. 1c). The selected ions are described in Table 2. After removing the ionsource fragment features, 5 metabolites were identified as nicotinamide, adenosine monophosphate, LysoPC (16: $0)$, LysoPC (18:0), and LysoPE (16:0) by comparing the MS/MS spectrum of each metabolite with that of the authentic compound. The levels of nicotinamide and adenosine monophosphate were significantly lower in 5xFAD mice than in WT mice (Fig. 2a and b), whereas the levels of LysoPC (16:0), LysoPC (18:0), and LysoPE
(16:0) were significantly higher $(q<0.05)$ in $5 x F A D$ mice than in WT mice at 8 or 12 months of age (Fig. 2c, d, and e).

Nicotinamide supplementation rescues the spine deficits in hippocampal primary neurons derived from 5xFAD mice Dendritic spine alteration is the cellular mechanism underlying neuronal activity and memory. It has been reported that treatment with nicotinamide rescues both short- and long-term memory impairment in 3xTg-AD mice, the triple-transgenic mice which harbor a knock-in mutation of PSEN1 $1_{\mathrm{M} 146 \mathrm{~V}}$, the Swedish double mutation of $\mathrm{APP}_{\mathrm{KM} 670 / 671 \mathrm{NL}}$, and a frontotemporal dementia mutation in tau (tau P301L $_{\text {L }}$ ) on a 129/C57BL/6 background [23].

Dendritic spine density in hippocampal primary neurons from 5xFAD mice was found to be significantly lower than hippocampal primary neurons from WT mice (WT + vehicle $[n=4$ ], $5.84 \pm 0.206$ [spine number/ $\mu \mathrm{m}] ; 5 \mathrm{xFAD}+$ vehicle $[n=3], 4.71 \pm 0.160$ [spine number $/ \mu \mathrm{m}], p<0.001$ ) (Fig. 3a and b). Next, we examined whether treatment with nicotinamide rescues the decrease in dendritic spine density in hippocampal primary neurons cultured from 5xFAD mice. Treatment with 10 $\mathrm{mM}$ nicotinamide for $24 \mathrm{~h}$ rescued the reduction in dendritic spine density in hippocampal primary neurons at 17-18 DIV (5xFAD + vehicle $[\mathrm{n}=3$ ], $4.71 \pm 0.160$ [spine number $/ \mu \mathrm{m}] ; \quad 5 \mathrm{xFAD}+10 \mathrm{mM}$ nicotinamide $[\mathrm{n}=3]$, $5.52 \pm 0.270$ [spine number $/ \mu \mathrm{m}$ ], $p<0.05$ ), while the same treatment did not affect dendritic spine density of WT hippocampal neurons (WT + vehicle [ $n=4], 5.84 \pm$ 0.206 [spine number $/ \mu \mathrm{m}$ ]; $\mathrm{WT}+10 \mathrm{mM}$ nicotinamide

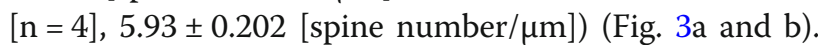
These findings indicate that reduced dendritic spine density in hippocampal primary neurons is, at least in part, due to the decreased nicotinamide concentration in the hippocampi from AD brains.

\section{Discussion}

$\mathrm{AD}$ is usually clinically diagnosed after its pathophysiological process has already started. In the early AD stage,

Table 2 Hippocampal biomarkers that were significantly different $(q<0.05)$ both in 8 and 12 months

\begin{tabular}{|c|c|c|c|c|c|c|c|c|c|}
\hline \multirow[t]{2}{*}{ Metabolites } & \multirow[t]{2}{*}{ Regulation $^{a}$} & \multicolumn{2}{|l|}{$8 \mathrm{M}$} & \multicolumn{2}{|l|}{$12 \mathrm{M}$} & \multirow{2}{*}{$\begin{array}{l}\text { Mass } \\
(\mathrm{m} / \mathrm{z})\end{array}$} & \multirow{2}{*}{$\begin{array}{l}\text { RT } \\
\text { (min) }\end{array}$} & \multirow{2}{*}{$\begin{array}{l}\text { lon } \\
\text { mode }\end{array}$} & \multirow[t]{2}{*}{ Fragment ion (m/z) } \\
\hline & & q-value & $p$-value & q-value & $p$-value & & & & \\
\hline Nicotinamide ${ }^{b}$ & down & 0.00161 & 0.00044 & 0.00161 & 0.00044 & 123.0554 & 0.97 & POS & $80.0502,78.0339,96.0439$ \\
\hline LysoPC $(16: 0)^{b}$ & up & $1.62 \mathrm{E}-05$ & $1.52 \mathrm{E}-06$ & 1.62E-05 & $1.52 \mathrm{E}-06$ & 496.3402 & 12.14 & POS & $184.0735,104.1076$ \\
\hline LysoPC $(18: 0)^{b}$ & up & 5.07E-07 & 1.09E-08 & 5.07E-07 & $1.09 \mathrm{E}-08$ & 524.3719 & 12.61 & POS & $184.0739,104.1074$ \\
\hline Phosphatidic acid ${ }^{c}$ & up & 2.01E-05 & $2.22 \mathrm{E}-06$ & 2.01E-05 & $2.22 \mathrm{E}-06$ & 487.2795 & 12.61 & POS & 445.2678 \\
\hline $\mathrm{AMP}^{\mathrm{a}}$ & down & 4.10E-06 & $8.02 \mathrm{E}-07$ & 4.10E-06 & $8.02 \mathrm{E}-07$ & 346.0545 & 0.97 & NEG & $96.9692,134.0466,150.9503$ \\
\hline LysoPE $(16: 0)^{c}$ & up & 1.67E-07 & $2.10 \mathrm{E}-08$ & 1.67E-07 & 2.10E-08 & 452.2761 & 11.98 & NEG & $255.2324,112.9855,196.0417$ \\
\hline LysoPE $(16: 0)^{\mathrm{a}}$ & up & $5.52 \mathrm{E}-08$ & 5.39E-09 & $5.52 \mathrm{E}-08$ & 5.39E-09 & 452.2772 & 12.1 & NEG & $255.2326,122.9857,196.0413$ \\
\hline LysoPE $(18: 0)^{c}$ & up & 1.37E-06 & 2.20E-07 & 1.37E-06 & 2.20E-07 & 480.3085 & 12.13 & NEG & 255.2322 \\
\hline
\end{tabular}

${ }^{a}$ Up- or down- regulation in 5xFAD vs. WT. ${ }^{\mathrm{b}}$ identified metabolite compared with authentic standard. ${ }^{\mathrm{C}}$ putative metabolites compared with database. $R T$ Retention time, CE Colision energy 
a
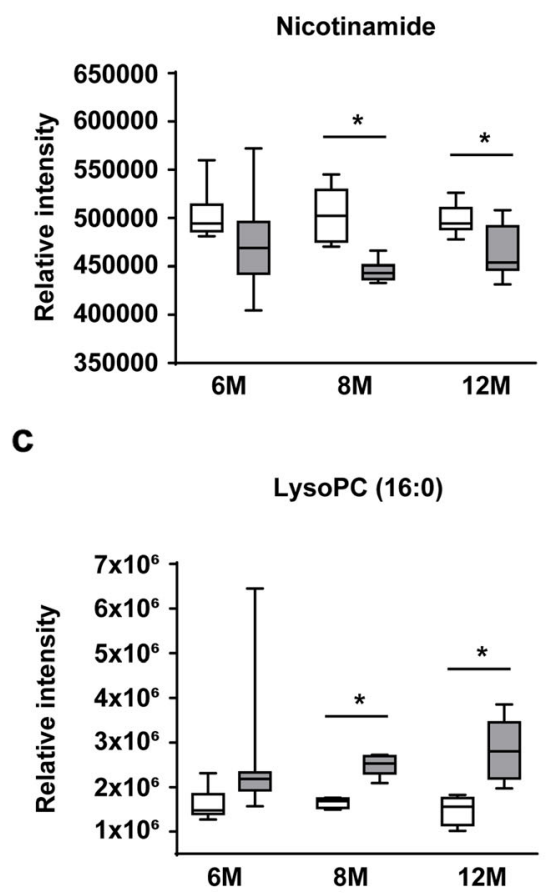

e b

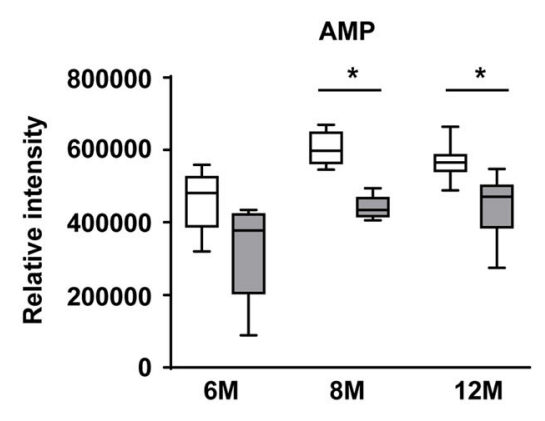

d

LysoPC (18:0)

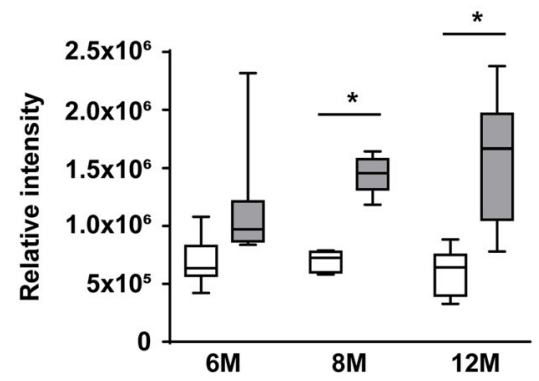

\section{LysoPE (16:0)}

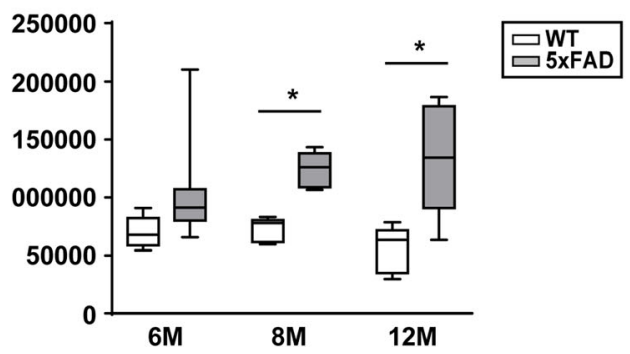

Fig. 2 Relative abundance of metabolites in the hippocampus in 5xFAD mice at each age. a nicotinamide, b AMP, c LysoPC (16:0), $\mathbf{d} L y$ soPC (18:0), and e LysoPE (16:0). Data are shown as means \pm SEM of at least 3 independent experiments; $6 \mathrm{M}-\mathrm{WT}(n=9), 6 \mathrm{M}-5 \mathrm{xFAD}(n=9), 8 \mathrm{M}-\mathrm{WT}$ $(n=6), 8$ M-5xFAD $(n=7), 12$ M-WT $(n=9)$, and 12 M-5xFAD $(n=8) .{ }^{*} q<0.05$ compared with age-matched WT mice. AMP, adenosine monophosphate; LysoPC, lysophosphatidylcholine; LysoPE, lysophosphatidylethanolamine

patients show mild cognitive impairment, which develops into $\mathrm{AD}$ in the rate of approximately $15 \%$ per year $[24,25]$. Therefore, the understanding of molecular pathogenesis in the pre-clinical process is essential for identifying treatment targets.

While there are some reports that have determined the metabolomics profiles associated with the progression of $\mathrm{AD}$ using postmortem brain tissues and blood samples [7, 26, 27], we are the first to address alterations in brain metabolism associated with $\mathrm{AD}$ in the hippocampus of $5 x F A D$ mice in three different disease stages.

Nicotinamide, an amide form of vitamin B3, is the primary precursor of nicotinamide adenine dinucleotide $\left(\mathrm{NAD}^{+}\right)$in mammalian cells [28, 29]. Nicotinamide is converted to $\mathrm{NAD}^{+}$through the activity of nicotinamide phosphoribosyltransferase, a rate-limiting enzyme in $\mathrm{NAD}^{+}$biosynthesis. As an energy substrate and cofactor for many enzymes, $\mathrm{NAD}^{+}$is critical for mitochondrial health and neuronal stress resistance $[30,31] . \mathrm{NAD}^{+}$is a crucial cofactor for cellular processes, such as glycolysis, fatty acid $\beta$-oxidation, tricarboxylic acid cycle, and DNA repair $[32,33]$. $\mathrm{NAD}^{+}$-dependent signaling, which is associated with neuronal development, survival, and function in the central nervous system, is implicated in neuroprotection [34]. The potential role of nicotinamide in AD has been highlighted in several studies. For instance, oral treatment with nicotinamide improves cognitive performance and reduces $A \beta$ and hyperphosphorylated tau pathologies in 3xTg-AD mice [34], and nicotinamide preserves cellular $\mathrm{NAD}^{+}$levels and increases the resistance of 


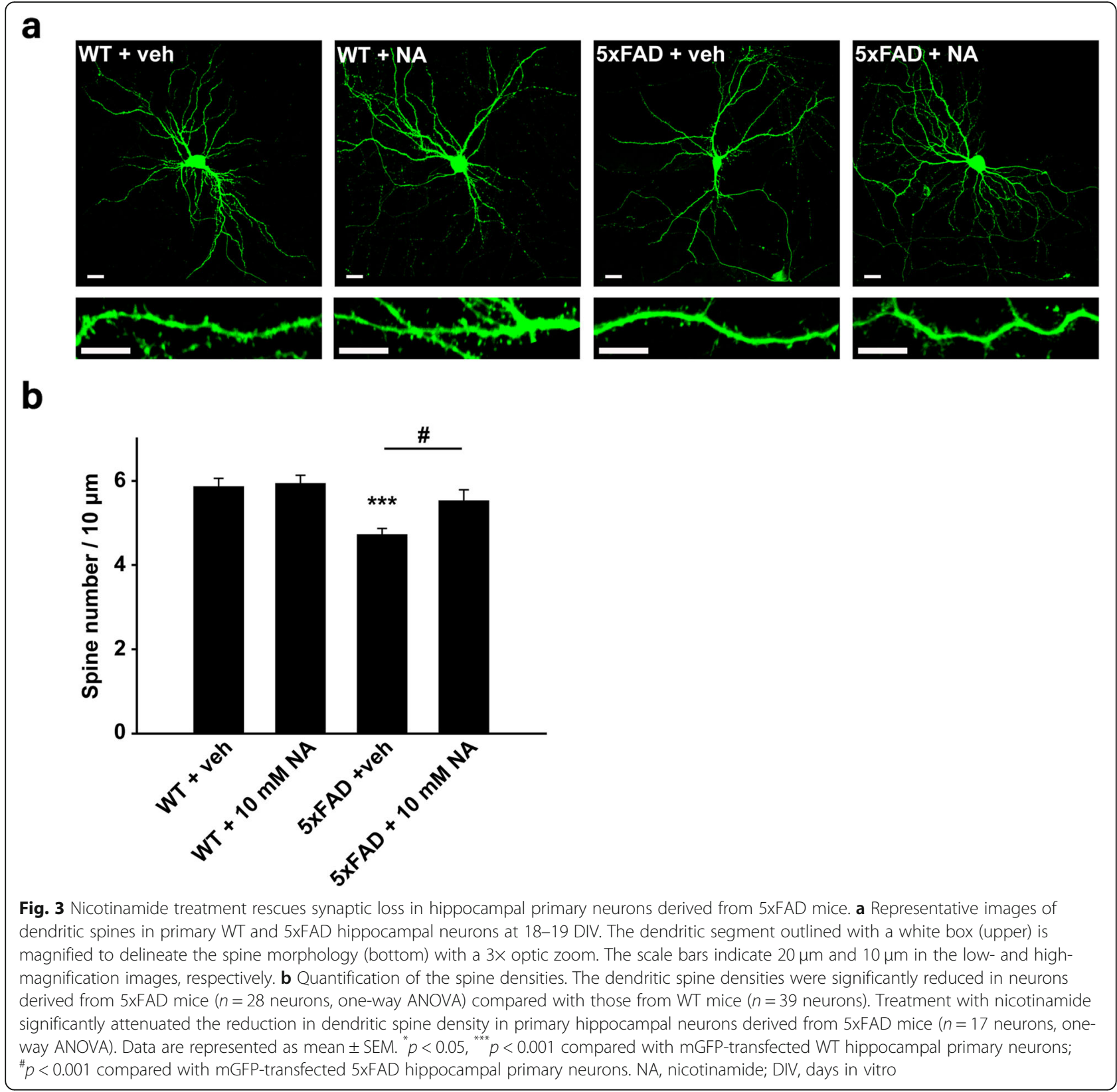

neurons against excitotoxicity [35]. The findings indicate the neuroprotective effect of nicotinamide and $\mathrm{NAD}^{+}$. However, no study has investigated the alterations of nicotinamide in the brain of $\mathrm{AD}$ models or patients. Although many reports have demonstrated the potential role of nicotinamide in neuroprotection and cognition, how the nicotinamide level changes in $\mathrm{AD}$ is not known. Here, we report novel findings that nicotinamide levels are decreased in the hippocampus of $5 \mathrm{xFAD}$ mice. This finding supports the hypothesis that NAD + -dependent signaling is disturbed in the $\mathrm{AD}$ brain.

In this study, we have investigated whether the supplementation of nicotinamide attenuates the reduction in dendritic spine density using cultured primary neurons from the hippocampus of 5XFAD mice (Fig. 3). Recent evidence has strongly indicated that cultured neurons from AD mouse models represent valuable models of this neurodegenerative disorder. Neurons derived from Tg2576 mice, a mouse model carrying a single mutation in human APP, show high intensity staining for the human APP protein/A $\beta$ fragments and increased vulnerability [36]. Additionally, the total number of dendritic spines, total spine extent, spine surface area, spine head diameter, and spine cross-sectional area are significantly decreased in neurons from APP/PS1 mice, a mouse model carrying double AD-associated mutations [37]. 


\section{Hippocampal metabolome (UPLC-QTQF) WT vs. 5xFAD}
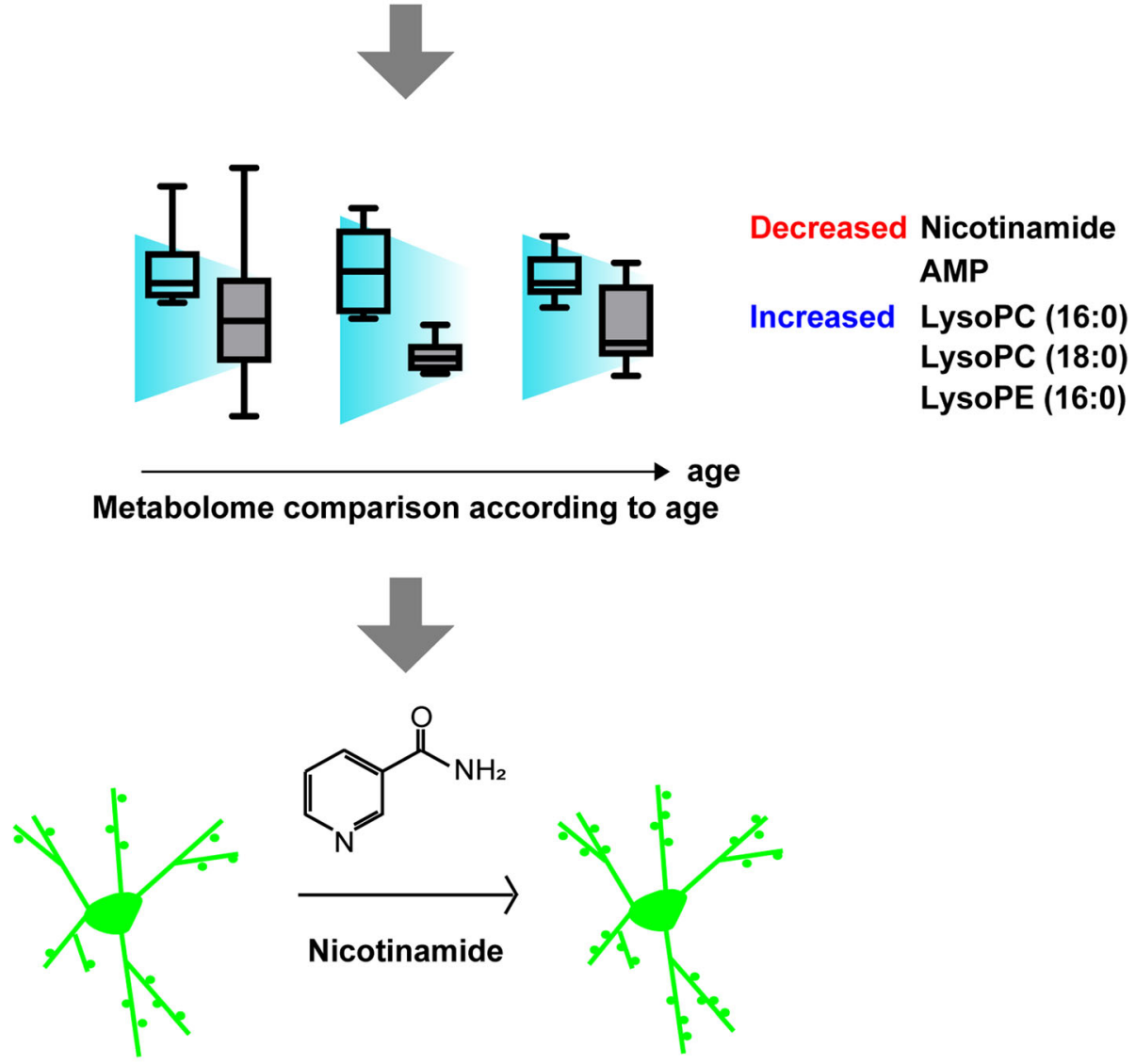

Hippocampal neuron from 5xFAD

Fig. 4 A graphical summary of this study, i.e. the reduction in nicotinamide in the hippocampus from 5xFAD mice as evaluated with untargeted metabolomics profiling, and the effect of supplementation on dendritic spine density of hippocampal primary neuron cultures from 5xFAD mice. 5xFAD mice displayed decreased content of nicotinamide and AMP, and increased content of LysoPC (16:0), LysoPC (18:0), LysoPE (16:0) in the hippocampus age-dependently. The addition of nicotinamide to hippocampal primary neuron culture from 5xFAD mice restored the reduced dendritic spine density. These results suggest nicotinamide as a therapeutic target in AD

Synaptic loss is one of the pathological hallmarks of AD and best correlates with cognitive decline, suggesting that it is a critical event in the pathophysiology of the disease [38]. Based on these previous reports, it can be said that cultured neurons from $\mathrm{AD}$ animal model reflect $A D$ phenotypes in vitro and can be used in $A D$ study. Thus, we assumed that the primary neurons from 5xFAD reflect the hippocampal pathophysiological characteristics of $5 \times \mathrm{xFAD}$ and treated nicotinamide to the primary neurons. Treatment with nicotinamide rescued synaptic deficits in hippocampal primary neurons derived from $5 \mathrm{xFAD}$ mice (Fig. 3). Together with those in the study by Liu et al. [34], our findings indicate that nicotinamide is a potential therapeutic drug for $\mathrm{AD}$. However, further studies are needed to investigate whether nicotinamide supplementation improves cognition in 5xFAD mice.

Reduced adenosine monophosphate levels have significant consequences, such as impairments in cellular energy homeostasis, because adenosine monophosphate plays a central role in glucose and lipid metabolism through the adenosine monophosphate-activated protein kinase, which is known to be decreased in $\mathrm{AD}$ brains $[39,40]$. In addition, significantly decreased adenosine monophosphate levels were detected in the hippocampus and cortex of APP/PS1 mice [13]. Notably, elevated adenosine monophosphate deaminase activity, which has been identified in the postmortem brain in AD patients, might lead to degradation of adenosine monophosphate and over-production of ammonia [41]. 
We found that lysoPC and lysoPE levels increased in the hippocampus in 5xFAD mice. Phospholipids, including lysoPC and lysoPE, are metabolized by phospholipase $\mathrm{A}_{2}\left(\mathrm{PLA}_{2}\right)$ from $\mathrm{PC}$ and $\mathrm{PE}$, respectively [42]. $\mathrm{PLA}_{2}$ activity has been shown to vary in different $\mathrm{AD}$ stages. In the early $\mathrm{AD}$ stage, $\mathrm{PLA}_{2}$ activity is decreased in the brains of $A D$ patients $[43,44]$. However, as the disease advances, $\mathrm{PLA}_{2}$ activity is elevated in $\mathrm{AD}$ brains [45]. Increased activation of $\mathrm{PLA}_{2}$ might induce an inflammatory condition by activating the arachidonic acid cascade, which plays a vital role in the inflammatory process. Furthermore, it has been demonstrated that LysoPC induces the formation of oligomer $A \beta$ and subsequent neurodegeneration in cultured neuronal cells [46]. Therefore, increased phospholipid levels might be associated with elevated PLA 2 activity and increased inflammation in AD brains.

\section{Conclusion}

This study is the first to investigate hippocampal metabolic markers in 5xFAD mice using an untargeted metabolomics approach. Another important aspect of this work is the comprehensive analysis across different disease progression stages. As summarized in the graphical summary of Fig. 4, we found decreased nicotinamide and adenosine monophosphate levels and increased LysoPC (16:0), LysoPC (18:0), and LysoPE (16:0) levels in the hippocampi in $5 \mathrm{xFAD}$ mice at 8 or 12 months of age. We also demonstrated that nicotinamide rescued the synaptic deficits of 5xFAD hippocampal primary neurons. This study is the first to demonstrate the reduced hippocampal nicotinamide levels in 5xFAD mice, and the findings suggest that the hippocampal nicotinamide level could be a useful therapeutic target for AD. Further studies are needed to clarify the metabolic pathway of nicotinamide and the molecular mechanism underlying altered nicotinamide levels in the hippocampus of 5 xFAD mice.

\section{Abbreviations}

5xFAD: Five human familial AD; AD: Alzheimer's disease; APP: Amyloid precursor protein; $A \beta$ : Amyloid $\beta$ peptide; DIV: Days in vitro; ESI: Electrospray ionization; FDR: False discovery rate; Lyso PC: Lysophosphatidylcholine; Lyso PE: Lysophosphatidylethanolamine; $\mathrm{NAD}^{+}$: Nicotinamide adenine dinucleotide; PLA 2 : Phospholipase $A_{2}$; PSEN 1: Presenilin-1; QC: Quality control; TCA: Tricarboxylic acid; WT: Wild type

\section{Acknowledgments}

Not applicable.

\section{Authors' contributions}

$\mathrm{HJ}-\mathrm{K}$ and BR-K performed most of the experiments and designed the project. HS-K and JY-C supervised the project, the manuscript and supporting funding acquisition. All authors read and approved the final manuscript.

\section{Funding}

This study was financially supported by the National Research Foundation of Korea (NRF) grant, funded by the Korean Government (2016R1A2B4012232) and also partly supported by Seoul National University Bundang Hospital
Research Fund, South Korea (02-2013-015). HJ.K. received a scholarship from the BK21-Plus Education Program provided by the National Research Foundation of Korea.

\section{Availability of data and materials}

Not applicable.

\section{Ethics approval and consent to participate}

All animal procedures were carried out following the National Institutes of Health Guidelines for the Humane Treatment of Animals, with approval from the Institutional Animal Care and Use Committee of Seoul National University (IACUC No. SNU-131016-1).

\section{Consent for publication}

Not applicable.

\section{Competing interests}

The authors declare that they have no competing interests.

\section{Author details}

${ }^{1}$ Department of Pharmacology, College of Medicine, Seoul National University, 103 Daehakro, Jongro-gu, Seoul, Republic of Korea. ${ }^{2}$ Department of Biomedical Sciences, College of Medicine, Seoul National University, 103 Daehakro, Jongro-gu, Seoul, Republic of Korea. ${ }^{3}$ Department of Clinical Pharmacology and Therapeutics, College of Medicine, Seoul National University, 103 Daehakro, Jongro-gu, Seoul, Republic of Korea. ${ }^{4}$ Kidney Research Institute, College of Medicine, Seoul National University, 103 Daehakro, Jongro-gu, Seoul, Republic of Korea. ${ }^{5}$ Seoul National University College of Medicine, Bundang Hospital, Bundang-Gu, Sungnam, Republic of Korea. ${ }^{6}$ Department of Pharmacology and Biomedical Sciences, Neuroscience Research Institute, College of Medicine, Seoul National University, 103 Daehakro, Jongro-gu, Seoul, Republic of Korea. ${ }^{7}$ Department of Clinical Pharmacology and Therapeutics, Seoul National University College of Medicine and Hospital, 101 Daehak-ro, Jongno-gu, Seoul 03080, Republic of Korea.

Received: 29 November 2019 Accepted: 4 February 2020

Published online: 07 February 2020

\section{References}

1. Gotz J, Ittner LM. Animal models of Alzheimer's disease and frontotemporal dementia. Nat Rev Neurosci. 2008:9(7):532-44.

2. Albert A, Borbely K. Molecular imaging of Alzheimer's disease. Orv Hetil. 2019:160(33):1289-95.

3. Oakley H, Cole SL, Logan S, Maus E, Shao P, Craft J, et al. Intraneuronal betaamyloid aggregates, neurodegeneration, and neuron loss in transgenic mice with five familial Alzheimer's disease mutations: potential factors in amyloid plaque formation. J Neurosci. 2006;26(40):10129-40.

4. Koh YH, Nguyen P, Osabutey AF, Choi BH, Kim AY. The regulation of neuroinflammation in brains of 5XFAD mice is underlying mechanisms of prevention of onset of Alzheimer's diseases by mature silkworm powders. Eur J Immunol. 2019:49:1434.

5. Hayashi K, Hasegawa Y, Takemoto Y, Cao C, Mukasa A, Kim-Mitsuyama S. Enhanced oxidative stress contributes to worse prognosis and delayed neurofunctional recovery after striatal intracerebral hemorrhage in 5XFAD mice. Eur J Neurosci. 2019. https://doi.org/10.1111/ejn.14596.

6. Hurtado MO, Kohler I, de Lange EC. Next-generation biomarker discovery in Alzheimer's disease using metabolomics - from animal to human studies. Bioanalysis. 2018:10(18):1525-46.

7. Varma VR, Oommen AM, Varma S, Casanova R, An Y, Andrews RM, et al. Brain and blood metabolite signatures of pathology and progression in Alzheimer disease: a targeted metabolomics study. PLoS Med. 2018;15(1): e1002482.

8. He X, Huang Y, Li B, Gong CX, Schuchman EH. Deregulation of sphingolipid metabolism in Alzheimer's disease. Neurobiol Aging. 2010;31(3):398-408.

9. Soderberg M, Edlund C, Alafuzoff I, Kristensson K, Dallner G. Lipid composition in different regions of the brain in Alzheimer's disease/senile dementia of Alzheimer's type. J Neurochem. 1992;59(5):1646-53.

10. Liu P, Fleete MS, Jing Y, Collie ND, Curtis MA, Waldvogel HJ, et al. Altered arginine metabolism in Alzheimer's disease brains. Neurobiol Aging. 2014; 35(9):1992-2003. 
11. van Gijsel-Bonnello M, Baranger K, Benech P, Rivera S, Khrestchatisky M, de Reggi $M$, et al. Metabolic changes and inflammation in cultured astrocytes from the 5xFAD mouse model of Alzheimer's disease: alleviation by pantethine. PLoS One. 2017;12(4):e0175369.

12. Gonzalez-Dominguez R, Garcia-Barrera T, Vitorica J, Gomez-Ariza JL. Regionspecific metabolic alterations in the brain of the APP/PS1 transgenic mice of Alzheimer's disease. Biochim Biophys Acta. 2014;1842(12 Pt A):2395-402.

13. Gonzalez-Dominguez R, Garcia-Barrera T, Vitorica J, Gomez-Ariza JL. Metabolomic screening of regional brain alterations in the APP/PS1 transgenic model of Alzheimer's disease by direct infusion mass spectrometry. J Pharm Biomed Anal. 2015;102:425-35.

14. Trushina E, Nemutlu E, Zhang S, Christensen T, Camp J, Mesa J, et al. Defects in mitochondrial dynamics and metabolomic signatures of evolving energetic stress in mouse models of familial Alzheimer's disease. PLoS One. 2012;7(2):e32737.

15. Schenck CA, Last RL. Location, location! Cellular relocalization primes specialized metabolic diversification. FEBS J. 2019. https://doi.org/10.1111/febs.15097.

16. Huang YR, Zhong C, Lin HX, Wang JY. A method for finding metabolic pathways using atomic group tracking. PLoS One. 2017;12(1):e0168725.

17. Trushina E, Mielke MM. Recent advances in the application of metabolomics to Alzheimer's disease. Biochim Biophys Acta. 2014;1842(8):1232-9.

18. Selkoe DJ, Hardy J. The amyloid hypothesis of Alzheimer's disease at 25years. EMBO Mol Med. 2016;8(6):595-608.

19. Nelson PT, Alafuzoff I, Bigio EH, Bouras C, Braak H, Cairns NJ, et al. Correlation of Alzheimer disease Neuropathologic changes with cognitive status: a review of the literature. J Neuropathol Exp Neurol. 2012;71(5):362-81.

20. Cruz-Sanchez FF, Girones X, Ortega A, Alameda F, Lafuente JV. Oxidative stress in Alzheimer's disease hippocampus: a topographical study. J Neurol Sci. 2010;299(1-2):163-7.

21. Berkowitz BA, Lenning J, Khetarpal N, Tran C, Wu JY, Berri AM, et al. In vivo imaging of prodromal hippocampus CA1 subfield oxidative stress in models of Alzheimer disease and Angelman syndrome. FASEB J. 2017;31(9):4179-86.

22. Benjamini $Y$, Hochberg Y. Controlling the false discovery rate - a practical and powerful approach to multiple testing. J Roy Stat Soc B Met. 1995;57(1): 289-300.

23. Green KN, Steffan JS, Martinez-Coria H, Sun XM, Schreiber SS, Thompson LM, et al. Nicotinamide restores cognition in Alzheimer's disease transgenic mice via a mechanism involving Sirtuin inhibition and selective reduction of Thr231-Phosphotau. J Neurosci. 2008;28(45):11500-10.

24. Petersen RC. Early diagnosis of Alzheimer's disease: is $\mathrm{MCl}$ too late? Curr Alzheimer Res. 2009;6(4):324-30.

25. Fiest KM, Roberts Jl, Maxwell CJ, Hogan DB, Smith EE, Frolkis A, et al. The prevalence and incidence of dementia due to Alzheimer's disease: a systematic review and meta-analysis. Can J Neurol Sci. 2016;43:S51-82.

26. Oresic M, Hyotylainen T, Herukka SK, Sysi-Aho M, Mattila I, Seppanan-Laakso T, et al. Metabolome in progression to Alzheimer's disease. Transl Psychiatry. 2011;1:e57.

27. Weng WC, Huang WY, Tang HY, Cheng ML, Chen KH. The differences of serum metabolites between patients with early-stage Alzheimer's disease and mild cognitive impairment. Front Neurol. 2019;10.

28. Belenky P, Bogan KL, Brenner C. NAD+ metabolism in health and disease. Trends Biochem Sci. 2007;32(1):12-9.

29. Kim HN, Li H, lyer S, Warren A, Manolagas S, Almeida M. The NAD(+) precursor nicotinamide riboside reverses the age-dependent decline of NAD(+) and Osteoprogenitor differentiation. J Bone Miner Res. 2017;32:S7.

30. Bonkowski MS, Sinclair DA. Slowing ageing by design: the rise of $N A D(+)$ and sirtuin-activating compounds. Nat Rev Mol Cell Biol. 2016;17(11):679-90.

31. Davila A, Liu L, Chellappa K, Redpath P, Nakamaru-Ogiso E, Paolella LM, et al. Nicotinamide adenine dinucleotide is transported into mammalian mitochondria. Elife. 2018;7:e33246.

32. Romani M, Hofer DC, Katsyuba E, Auwerx J. Niacin: an old lipid drug in a new NAD+ dress. J Lipid Res. 2019;60:741-6.

33. Zhang N, Sauve AA. Regulatory effects of $N A D(+)$ metabolic pathways on Sirtuin activity. Prog Mol Biol Transl. 2018;154:71-104

34. Liu D, Pitta $M$, Jiang $H$, Lee $J H$, Zhang $G$, Chen $X$, et al. Nicotinamide forestalls pathology and cognitive decline in Alzheimer mice: evidence for improved neuronal bioenergetics and autophagy procession (vol 34, pg 1564, 2013). Neurobiol Aging. 2013;34(9):E3.

35. Liu D, Gharavi R, Pitta M, Gleichmann M, Mattson MP. Nicotinamide prevents $\mathrm{NAD}(+)$ depletion and protects neurons against excitotoxicity and cerebral ischemia: $\mathrm{NAD}(+)$ consumption by SIRT1 may endanger energetically compromised neurons. Neuromolecular Med. 2009;11(1):28-42.
36. Baldassarro VA, Marchesini A, Giardino L, Calza L. Vulnerability of primary neurons derived from Tg2576 Alzheimer mice to oxygen and glucose deprivation: role of intraneuronal amyloid-beta accumulation and astrocytes. Dis Model Mech. 2017;10(5):671-8.

37. Kommaddi RP, Das D, Karunakaran S, Nanguneri S, Bapat D, Ray A, et al. A beta mediates F-actin disassembly in dendritic spines leading to cognitive deficits in Alzheimer's disease. J Neurosci. 2018;38(5):1085-99.

38. de Wilde MC, Overk CR, Sijben JW, Masliah E. Meta-analysis of synaptic pathology in Alzheimer's disease reveals selective molecular vesicular machinery vulnerability. Alzheimers Dement. 2016;12(6):633-44.

39. Cai ZY, Yan LJ, Li KS, Quazi SH, Zhao B. Roles of AMP-activated protein kinase in Alzheimer's disease. Neuromolecular Med. 2012;14(1):1-14

40. Feng HC, Wang CM, He W, Wu XJ, Li SJ, Zeng ZK, et al. Roflumilast ameliorates cognitive impairment in APP/PS1 mice via CAMP/CREB/BDNF signaling and anti-neuroinflammatory effects. Metab Brain Dis. 2019;34(2):583-91.

41. Sims B, Powers RE, Sabina RL, Theibert AB. Elevated adenosine monophosphate deaminase activity in Alzheimer's disease brain. Neurobiol Aging. 1998;19(5):385-91.

42. Steinbrecher UP, Parthasarathy S, Leake DS, Witztum JL, Steinberg D. Modification of low-density lipoprotein by endothelial-cells involves lipidperoxidation and degradation of low-density lipoprotein phospholipids. P Natl Acad Sci-Biol. 1984:81(12):3883-7.

43. Schaeffer EL, Forlenza OV, Gattaz WF. Phospholipase A (2) activation as a therapeutic approach for cognitive enhancement in early-stage Alzheimer disease. Psychopharmacology. 2009;202(1-3):37-51.

44. Mury FB, Barbosa NR, Defillipo PP, Mendes CT, Gattaz WF, Dias-Neto E. Memantine induces expression of PLA2 genes in rat brain: possible implications for reverse learning and memory of Alzheimer's disease patients. Eur Psychiatry. 2008;23:S292-3.

45. Stephenson DT, Lemere CA, Selkoe DJ, Clemens JA. Cytosolic phospholipase A2 (CPLA2) immunoreactivity is elevated in Alzheimer's disease brain. Neurobiol Dis. 1996;3(1):51-63.

46. Sheikh AM, Michikawa M, Kim SU, Nagai A. Lysophosphatidylcholine increases the neurotoxicity of Alzheimer's amyloid beta (1-42) peptide: role of oligomer formation. Neuroscience. 2015;292:159-69.

\section{Publisher's Note}

Springer Nature remains neutral with regard to jurisdictional claims in published maps and institutional affiliations.

Ready to submit your research? Choose BMC and benefit from:

- fast, convenient online submission

- thorough peer review by experienced researchers in your field

- rapid publication on acceptance

- support for research data, including large and complex data types

- gold Open Access which fosters wider collaboration and increased citations

- maximum visibility for your research: over $100 \mathrm{M}$ website views per year

At BMC, research is always in progress.

Learn more biomedcentral.com/submission 